\title{
One-Pot Synthesis of Heterometallic Copper-Manganese Complexes with Polyfunctional Imidoyl Amidine Ligands
}

\author{
R Castañeda ${ }^{1}$, M Rouzières $^{2}$, R Clérac $^{2}, \mathrm{~J} \mathrm{Brusso}^{3}$ \\ ${ }^{1}$ University of Ottawa, Ottawa, ${ }^{2}$ Université du Bordeaux, Pessac, FM, ${ }^{3}$ Department of Chemistry \\ and Biomolecular Sciences, University of Ottawa, Ottawa, Ontario \\ lcast029@uottawa.ca
}

Bidentate ligands such as acetylacetonate (acac) and 1,3-diketoiminate (nacnac), or tridentate ligands such as terpyridine (terpy), are often employed to isolate metallic complexes for a diverse range of applications including water splitting, organic catalysis, molecular magnets and photosensitizers to name a few. In that regard, the N-2pyrimidylimidoyl-2-pyrimidylamidine (PmImAm) ligand is an excellent candidate for the development of coordination complexes as it possesses both a bidentate coordination site similar to acac and nacnac, as well as a tridentate terpy like coordination site. Having these two types of coordination environments within the same complex provides an avenue to explore polynuclear mixed metal complexes. While heterometallic complexes are typically prepared in a step-wise manner by first isolating a mononuclear complex then using it as a starting material to coordinate a second metal ion, by taking advantage of the two distinctive coordination sites in PmImAm we have achieved heterometallic copper-manganese complexes via a one-pot synthesis. Furthermore, through ppm level control over the degree of hydration (e.g., $300 \mathrm{ppm}$ vs. $10000 \mathrm{ppm}$ ), the nuclearity and topology of these polynucelar heterometallic complexes can be tailored. This presentation will describe both a trinuclear complex with a central square planar $\mathrm{Cu}(\mathrm{II})$ ion coordinated in the bidentate pocket of PmImAm, and two peripheral pentacoordinate $\mathrm{Mn}$ (II) ions in the terpy-like site, as well as a hexanuclear complex with four penta-coordinate $\mathrm{Mn}$ (II) ions and two penta-coordinate $\mathrm{Cu}$ (II) ions. This presentation will focus on the synthesis and structural analysis of these complexes via single crystal X-ray diffraction, as well as their magnetometry studies.

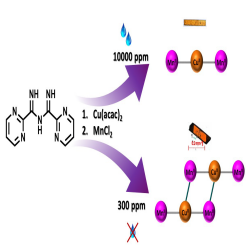

Figure 1

Acta Cryst. (2020). A76, a19 